\title{
Simulation of an Improved Microactuator with Discrete MSM Elements
}

\author{
Berta Spasova and Hans H. Gatzen \\ Institute for Microtechnology, Center for Production Technology, Leibniz Universitaet Hannover, An \\ der Universitaet 2, 30823 Garbsen, Germany \\ spasova@imt.uni-hannover.de
}

Keywords: MSM microactuator, NiMnGa, FEM simulation.

\begin{abstract}
Magnetic Shape Memory (MSM) alloys are a new class of "smart" materials. In the martensite state, they exhibit a reversible strain due to a reorientation of twin variants, based on twin boundary motion driven by an external magnetic field occurring in the martensite state. This effect allows for the development of linear microactuators.

This work presents the simulation results for the fabrication of a microactuator based on an MSM alloy with an optimized design. A stator element consists of a NiFe45/55 flux guide, two poles, and double-layer $\mathrm{Cu}$ coils wound around each pole for generating the magnetic field. The MSM material applied is NiMnGa. The integrated microactuator is subjected to dynamic simulation, using a "checkerboard" pattern to locally switch the magnetic properties when the relative permeability $\mu_{r}$ is changed. The model is described with the Ansys Parametric Design Language (APDL). Design, modeling, and simulation of the magnetic system including MSM material, are conducted by Finite Element Method (FEM) analysis using the software tool ANSYSTM.
\end{abstract}

\section{Introduction}

Magnetic Shape Memory (MSM) alloys are recognized as promising and high performance materials in the field of Micro Electro-mechanical System (MEMS) applications. The recent progress in designing a new class of MSM alloys is based on the martensite-martensite twin boundary motion driven by a magnetic field [1]. Material exposed to an external magnetic field shows a Magnetic Field Induced Strain (MFIS). The field induced martensite twin reorientation is possible in materials with high magnetocrystalline anisotropy energy (MEA) and low energy of twin boundary motion. With 5 to 10 percent, the MFIS observed in MSM alloys is substantial, which allows to using this effect in linear microactuators.

Several research groups reported on a 6 percent magneto-strain effect in a five-layer modulated tetragonal martensite phase [2] as well as in a ten-layer modulated martensite phase [3]. The maximal MFIS (up to 10 percent) was observed by a seven-layer modulated orthorhombic martensite crystal [4]. Several kinds of martensite materials exhibit a MFIS, for example NiMnGa [5, 6, 7], FePt [8], and FePd [9].

For the investigation of a microactuator with discrete MSM elements, the Institute for Microtechnology (imt) at the Leibniz Universitaet Hannover received MSM stripes (NiMnGa) from the Hahn-Meitner Institute in Berlin. The samples feature a relative permeability $\mu_{r}$ of 2 before the reorientation and a permeability $\mu_{r}$ of 6 after the reorientation. Based on these data, a microactuator was simulated and optimized. In the first stage, the magnetic system was designed and modelled using the Finite Element Method (FEM). The simulations are executed applying the software tool ANSYS $^{\text {TM }}$. FEM analyses were used to find out the optimal design of the magnetic micro system by changing the magnetic properties of the MSM material. The optimal microactuator consists of pairs of U-shaped thin-film cores, with each pole carrying a thin-film coil. The coil system consists of a double-layer coil, where the top coil straddles the left pole and the bottom coil the right pole. The fabrication steps and characterization of the optimal microactuator with discrete NiMnGa MSM bulk material is presented in [10].

This paper describes the 2-D and 3-D modeling as well as the simulation results of an improved MSM microactuator using thin-film technology for the stator fabrication and discrete MSM stripes as actuating element. The goal of these simulations is to maximize the fraction of the 
microactuator's cross section exposed to the external magnetic field. In this model, the coil system consists of a left and right double-layer coil with two turns each. The improved design differs from the first research stage in the coil arrangement. By straddling the left and the right coil around the left and the right pole, respectively, the exposed fraction of the cross section can be increased. Furthermore, a dynamic modeling of the improved microactuator was conducted. To model the local switch of the magnetic properties after a change between the MSM material's twin variants, a "checkerboard" pattern was used. It allows to locally allocating the material's relative permeability $\mu_{r}$

\section{Modeling of Magnetic Microsystems}

Miniaturized magnetic actuators are key components in micro systems. Microactuation based on the electromagnetic principle provides rather high forces, high frequencies, and features a low driving voltage [11]. Optimizing the fraction of the cross section exposed to the external magnetic field in a magnetic microactuator is a key requirement for a highly efficient microactuator. For designing an improved microactuator, complex electromagnetic simulations are needed. An approach to design magnetic microactuators with discrete MSM elements is shown in Fig. 1. The magnetic microactuator with integrated MSM elements consists of a NiFe45/55 flux guide, two poles, and double-layer $\mathrm{Cu}$ coils wound around each pole for generating the magnetic field. For flux guides, a magnetic material with a high permeability $\mu_{r}$ (featuring a low magnetic reluctance) is used while the air gap represents an element with a great reluctance typically required to create a force and motion.

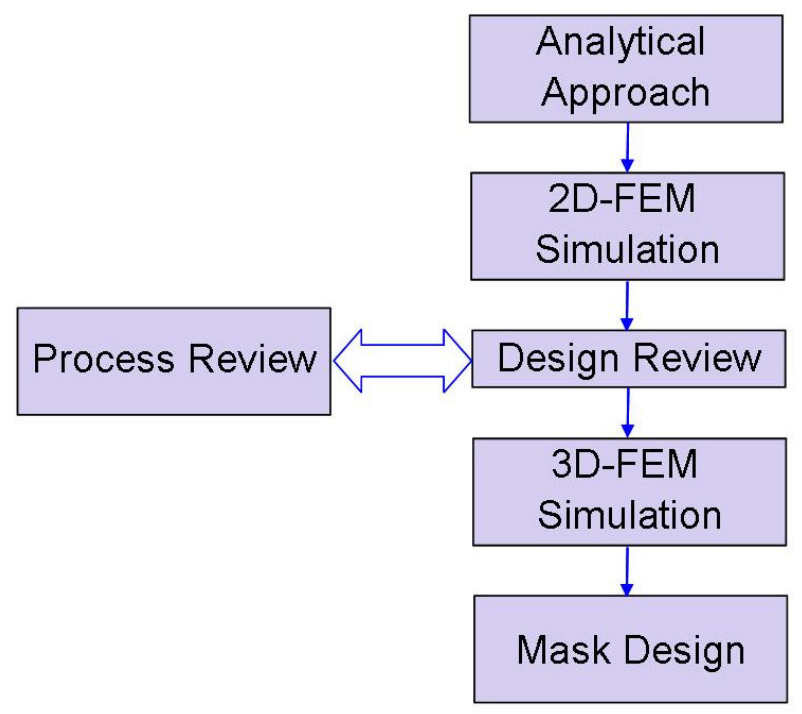

Fig. 1: Approach of modeling and simulation of magnetic microactuators

The first step of modeling magnetic microsystems is an analytical approach for defining a preliminary design with an analysis of the components. In our case, the components of the excitation system are a NiFe45/55 flux guide and $\mathrm{Cu}$ coils as the basic elements. The air gap between the basic elements and the discrete MSM elements is $5 \mu \mathrm{m}$. The width of the MSM stripes is $100 \mu \mathrm{m}$. Each system consists of double-layer spiral coils with 2 x 2 turns featuring an aspect ratio of 1.5 to 1 . All geometries are presented in Table 1 and in Fig. 2. The basic actuator components are joined to create a complete actuator and a magnetic circuit. 
Table 1: Geometry of the improved microactuator

\begin{tabular}{|l|l|}
\hline \multicolumn{2}{|l|}{ Flux guide } \\
\hline Length of the magnetic core: $a$ & $360 \mu \mathrm{m}$ \\
\hline Thickness of the flux guides: $b$ & $40 \mu \mathrm{m}$ \\
\hline Pole width: $c$ & $100 \mu \mathrm{m}$ \\
\hline Pole height: $d$ & $71.5 \mu \mathrm{m}$ \\
\hline Coil system & $23.5 \mu \mathrm{m}$ \\
\hline Coil width & $35 \mu \mathrm{m}$ \\
\hline Coil height & $10 \mu \mathrm{m}$ \\
\hline Distance between turns & $0.5 \mu \mathrm{m}$ \\
\hline Vertical distance between the coil-layer &
\end{tabular}

The next step was the generation of an FEM model. Fig. 2 shows the 2-D model of the MSM actuator used for the simulations. Table 2 presents the material properties for all dimensions that where used in the FEM simulations.

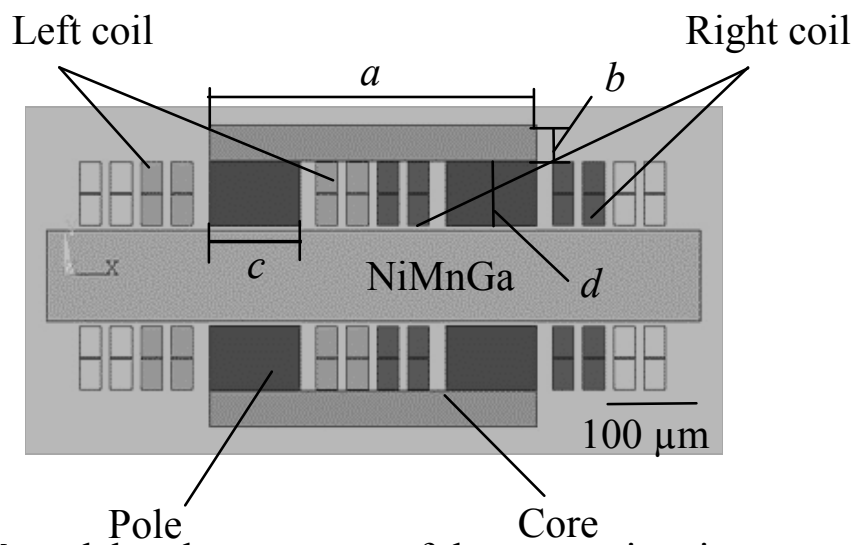

Fig. 2: ANSYSTM model and parameters of the magnetic microactuator using NiMnGa stripes as discrete element

Table 2: Material properties of the microactuator components used for the ANSYSTM simulations

\begin{tabular}{|l|l|l|}
\hline \multicolumn{1}{|c|}{ Material } & \multicolumn{1}{|c|}{ Component } & Relative permeability $\boldsymbol{\mu}_{\boldsymbol{r}}$ \\
\hline $\mathrm{Cu}$ & Coil & 1 \\
\hline $\mathrm{NiFe} 45 / 55$ & Flux guide & $80 / 100($ pole/core $)$ \\
\hline $\mathrm{MSM}$ alloy $(\mathrm{NiMnGa})$ & Actuating element & 2 \\
\hline
\end{tabular}

The microactuator with discrete MSM elements was simulated using the software tool ANSYSTM. Applying an MSM material, the critical magnetic field strength $H_{c r i t}$ is an important parameter for the simulations. For NiMnGa, the magnetic field $H_{c r i t}$ to initiate a switch between the twin variants is $50 \mathrm{kA} / \mathrm{m}$. $H_{\text {crit }}$ was determined by Vibrating Sample Magnetometer (VSM) measurements [12]. During the whole actuation process, the material remains in a martensitic state. For the stator coils, a nominal current density $J$ in impulse mode of $2.0 \times 10^{9} \mathrm{~A} / \mathrm{m}^{2}$ is selected and applied. This value is dictated by the current carrying capability for the selected micro coil fraction of the cross section. Fig. 3 depicts 2-D simulation results, comparing the initial and the improved design. While the initial design only reached the critical field strength $H_{\text {crit }}$ in 60 percent of the fraction of the cross section, the fraction of the cross section for the improved design is 67 percent. 
An increase of 7 percent represents a considerable improvement achieved with the optimized microactuator.

a)

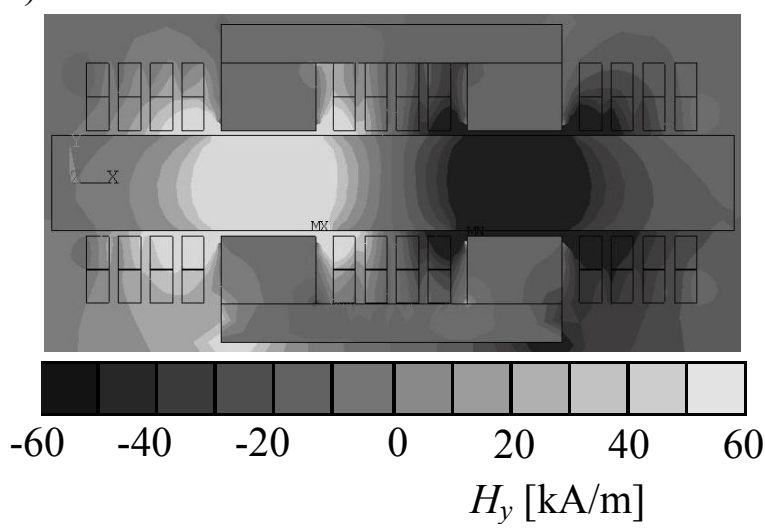

b)

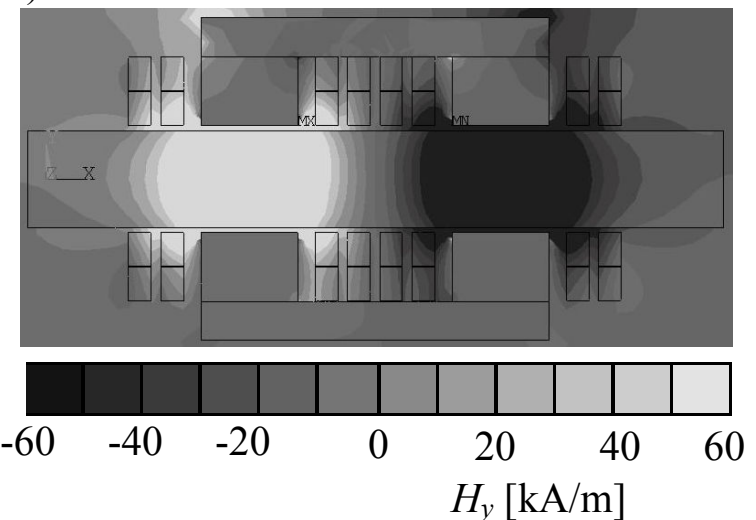

Fig. 3: Results of 2-D model, $H_{y}$ for a nominal current density $J$ of $2 \times 10^{9} \mathrm{~A} / \mathrm{m}^{2}$ : a) in the initial version of the MSM microactuator; $b$ ) in the optimized MSM microactuator

To further investigate the microactuator behavior, a 3-D simulation was conducted. The 3-D simulation yields less favorable conditions than the 2-D simulation, which is typical. For the original design, the fraction of the cross section reaching the critical field strength $H_{\text {crit }}$ is 40 percent [13]. For the new optimized design, the fraction of the cross section increases to 48 percent. Fig. 4 illustrates the simulation results.

a)
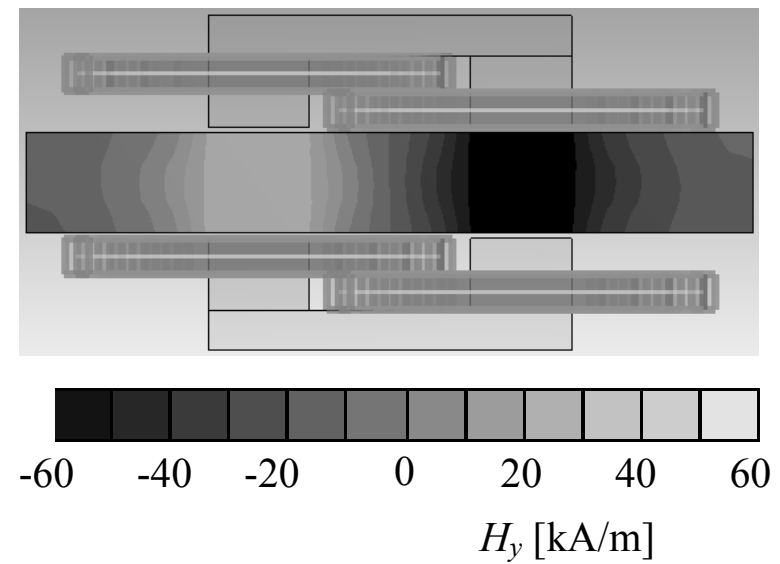

b)
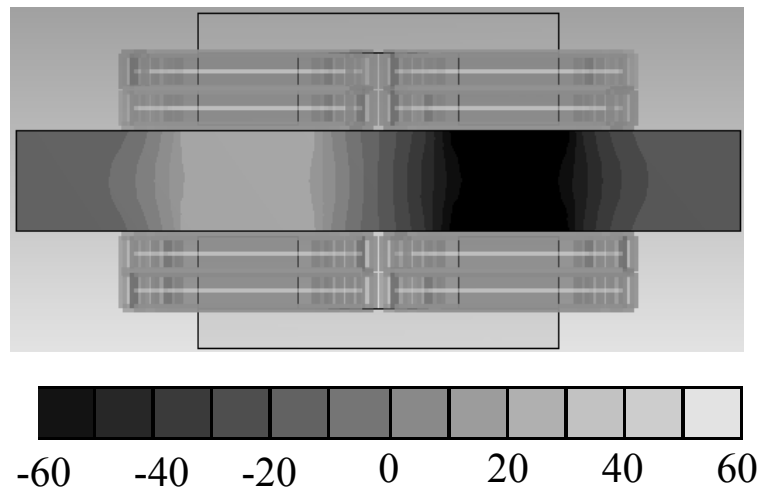

$H_{y}[\mathrm{kA} / \mathrm{m}]$

Fig. 4: Results of 3-D model, $H_{y}$ for a nominal current density $J$ of $2 \times 10^{9} \mathrm{~A} / \mathrm{m}^{2}$ : a) in the initial version of the MSM microactuator; $b$ ) in the optimized MSM microactuator

\section{Dynamical Simulation}

The presented dynamical simulation of the improved microactuator with discrete MSM elements will be used to calculate approximately the mechanical response (elongation of the actuator) as function of the time increasing the magnetic field. It was conducted with the Ansys Parametric Design Language (APDL). The first tool of the ANSYS ${ }^{\mathrm{TM}}$ levels is a preprocessor, which executes the process modeling, defines the material properties, and generates the finite element model. Building a finite element model requires to define the element types, material properties, and the model geometry. For the simulation, PLANE13 (2-D coupled-field solid) is used as element type. The element has nonlinear magnetic capabilities for modeling the $B-H$ curve, the relative permeability $\mu_{r}$, and the demagnetization curves. After defining the element type, the material properties were determined. As mentioned before, for allowing a local allocation of magnetic 
properties, a "checkerboard" system was used, it consists of 30 quadrants. For any of the "checkerboard" fields, the relative permeability $\mu_{r}$ can be chosen individually, thus representing the MSM material's actual state. The next step was defining the boundary conditions of the model. As requirements of an improved microactuator from major preference is providing a sufficient magnetic field $H_{\text {crit }}$ (necessarily $50 \mathrm{kA} / \mathrm{m}$ for NiMnGa) in vertical direction in the MSM area to achieve a switch from one twin variant to the other. This field, generating a reorientation of the MSM material was determined. Next, the model was solved and the results were postprocessed. Of particular interest is to implement a new simulation method indicating the change of the magnetic properties. This approach allows to change the relative permeability $\mu_{r}$ of the MSM material when switching between the twin variants. In this case, the magnetic permeability $\mu_{r}$ in the unmagnetized state is changed by the simulation tool automatically, as soon as the critical magnetic field $H_{c r i t}$ in the exposed area of the MSM material is exceeded. The simulations show, that in a defined area of the MSM "checkerboard", the exposed area grows. In this case, the domain in the material expanding due to the external magnetic field is arranged in all areas. In the last step of the dynamic simulations, the field strength $H$ exceeds $H_{\text {crit }}$ in all quadrants. Finally, the results can be observed and they can be plotted. The algorithm to calculate the dynamic simulations is assembled with APDL. The argument in ANSYS ${ }^{\mathrm{TM}}$ commands uses arithmetic equations and functions. The ANSYS $^{\text {TM }}$ software tool works with FORTRAN ${ }^{\text {TM }}$ functions. In this case, the model is generated and solved automatically. Fig. 5 depicts the results with all steps of the dynamical simulations.

a)

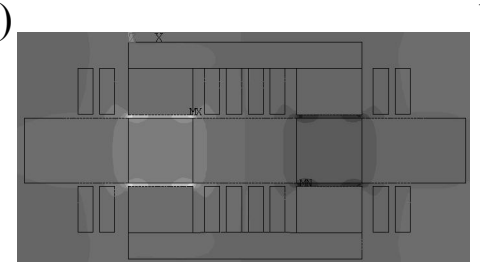

d)

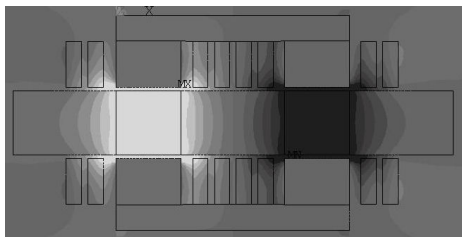

b)

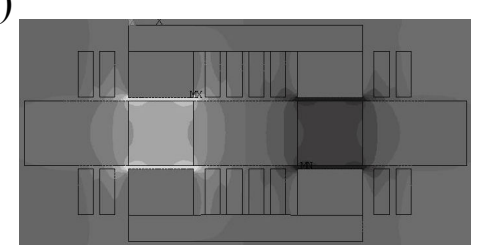

c)

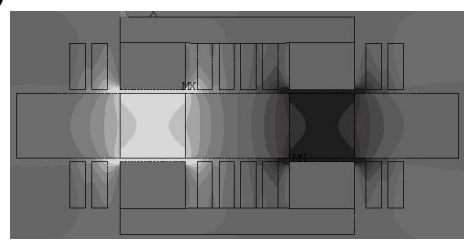

e)

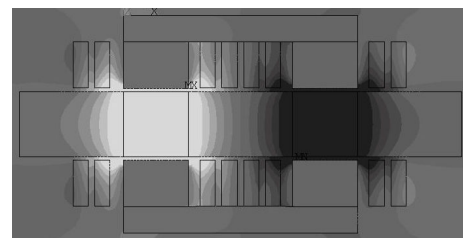

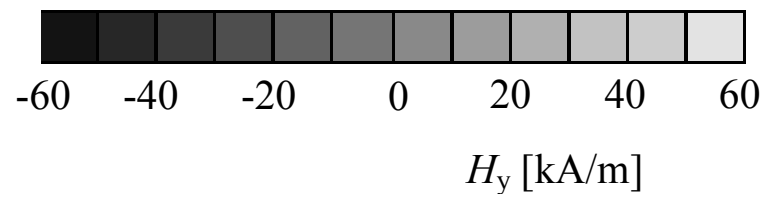

Fig. 5: $H_{y}$ as function of the current: a) $20 \%$, b) $40 \%$, c) $60 \%$, d) $80 \%$, and e) $100 \%$ of the maximum current

\section{Conclusions}

Previous work has demonstrated the importance of a dynamic FEM analysis step for the microactuator design. The main challenge was to determine if a sufficient magnetic field strength required for the change from one twin variant to the other (for NiMnGa $50 \mathrm{kA} / \mathrm{m}$ ) was reached. The next goal was to maximize the fraction of the cross section of the MSM element exposed to the magnetic field, which was accomplished. Using a 3-D simulation, an increase of the fraction of the cross section exposed to an external magnetic field for the optimized microactuator using NiMnGa as MSM element from 40 percent to 48 percent could be accomplished. Furthermore, by choosing a dynamical approach for the simulation of magnetic microactuators, a local change in magnetic properties could be modeled. 


\section{Acknowledgement}

This work was sponsored in part by the DFG (German Research Foundation) within the Priority Programme SPP 1239 "Magnetic Shape Memory".

\section{References}

[1] A. Sozinov, A.A. Likhachev, and K. Ullakko, IEEE Trans. on Magn, Vol. 38 (2002), pp. 2814

[2] R. Tickle, R.D. James, T. Shield, M. Wuttig, and V.V. Kokorin, IEEE Trans. on Magn, Vol. 35 (1999), pp. 4301

[3] S.J. Murray, M. Marioni, S.M. Allen, R.C. O’Handley, and T.A. Lograsso, Appl. Phys. Lett., Vol.77 (2000), pp. 886

[4] A. Sozinov, A.A. Likhachev, N. Lanska, and K. Ullakko, Appl. Phys. Lett., Vol. 80 (2002), pp. 1746

[5] I. Takeuchi, O.O. Famodu, J.C. Read, M.A. Aronova, K.S. Chang, and C. Craciunescu, Nat. Mater. Vol. 2 (2003), pp. 180

[6] S.J. Murray, M.A. Marioni, A.M. Kukla, J. Robinson, R.C. O’Handley, and S.M. Allen, J. Appl. Phys. Vol. 87 (2000), pp. 5774

[7] M.A. Marioni, R.C. O'Handley, S.M. Allen, S.R. Hall, D.I. Paul, and M.L. Richard, J. Magn. Magn. Mate. Vol. 35 (2005), pp. 290

[8] R.D. James, and M. Wuttig, Philos. Mag. A. Vol. 77 (1998), pp. 1273

[9] T. Kakeshita, T. Takeuchi, T. Fukuda, T. Saburi, R. Oshima, and S. Muto, Mater. T. JIM. Vol. 41 (2000), pp. 882

[10] B. Spasova, M.C. Wurz, and H.H. Gatzen, Prog. Actuator 2008, $11^{\text {th }}$ Conference on new Actuators, Bremen, Germany (2008), pp. 713

[11] D. Dinulovic and H.H. Gatzen, Prog. EuroSimE 2008, Freiburg, Germany (2008), pp. 217

[12] B. Spasova, M. Wurz, and H. H. Gatzen, EUROMAT 2007, Nürnberg, Germany, Physica Status Solidi (a), Vol. 10 (2008), pp. 2307

[13] B. Spasova, M.C. Wurz, and H.H. Gatzen, Prog. E-MRS-Symposium 2007, Warshaw, Poland, (2008), pp. 243 